\title{
Co-Simulation Study for Fuzzy ESP Control Strategy on Vehicle
}

\author{
Shengqin $\mathrm{Li}^{*}$, Li Zhao and Chunbo Yang
}

Traffic College, Northeast Forest University, Harbin, 150040, China

\begin{abstract}
ESP can help the road vehicle to improve driving dynamics and prevent accidents which result from loss of control. Based on the theory and account method of Multi-body System Dynamics, the Multi-body System Dynamics model of an A-class car is built by the ADAMS/Car software, which includes steering system, front and rear suspension, braking system, body, and so on. In order to study the handling stability of vehicle with ESP control system, the vehicle yaw rate is set as the controlling aim, to build the fuzzy controller of ESP. The performance evaluation methods of vehicle with ESP are proposed to evaluate the handling stability of vehicle with ESP. The results show that the vehicle with ESP can keep the right path, the yaw rate and the vehicle slip angle is all controlled better. The handling stability of vehicle can be improved.
\end{abstract}

Keywords: Simulation, ESP, fuzzy control, intelligent transportation, co- virtual prototype.

\section{INTRODUCTION}

ESP (Electronic Stability Program)can help to control the vehicle's yaw rate and limit the side-slip angle in a certain range by controlling the wheel braking force or the engine output torque, to avoid sudden obstacles ahead, u-turn lane in high-speed or under extreme adhesion conditions, it can assist the driver to control the vehicle handling and stability to prevent the vehicle from understeering or oversteering, rollover risk condition [1]. According to statistics, fatal accidents of the vehicle with this system decreased from $30 \%$ to $50 \%$, while such accidents of the SUV reduced by $50 \%-70 \%$. If all vehicles in the U.S. were installed with ESP, it would reduce 600,000 accidents and 10,000 fatal accidents each year.

ESP can reduce the incidence of the car accident significantly. Researchers at the University of IOWA indicate that DSC can reduce the chances of a malfunction of the driver in emergency [2]. Research on 1.5 million Mercedes-Benz cars showed that, the proportion of accidents decreased by $30 \%$ [3]. Toyota have analyzed one million cases of driving accidents, the results showed that, driving accidents decreased by $35 \%$ after using DSC, and $50 \%$ reduction in the number of serious injury [4]. Because of the DSC significant contribution in the field of automotive active safety, it has been accepted by consumers in a wide range, and is being popular from caravan and SUV to midrange cars. NHTSA provides that all the car under 4.5 million tons should be installed with ESP [5].

ESP system is constituted by increasing the AYC system on the basis of the ABS/TCS. AYC can apply the activating brake on the four wheels, and generate a yaw moment of couple on the vehicle, improve the dynamic stability of the vehicle in the steering process. Since 1988, after the civil ABS (1978) and TCS (1986) achieved mass production in the Bosch company, Heess, described a kind of vehicle dynamics control system integrated ABS, TCS, suspension system and steering system, and proposed the corresponding industrial solutions [6]. Inspired by this, under the leadership of A.T. van Zanten and Armin Mueller, Bosch and Mercedes-benz company developed the ESP, and matched to the Mercedes-Benz S600 sedan successfully. At the same time, BMW and TEVES company also carried out the research of ESP technology, but different manufacturers named the ESP with their own characteristics [7]. Now there are 7 auto parts suppliers produced ESP system, such as Bosch, Denso, Teves, Delphi, Aisin Seiki, TRW and Mando, in which Bosch company's total sales have been more than 10 million units by the end of 2003 .

Since the ESP study needs long cycle and large investment, most of research institutions mainly focus on the simulation. In the paper, the ESP simulation model of a car is established and to conduct research the control strategy of ESP. Test and simulation analysis are proposed in condition of dry road and ice road to verify the control strategy.

\section{ESTABLISHMENT OF VIRTUAL PROTOTYPE MODEL}

Using the multi-body dynamic model of vehicle, establishing suspension and steering system by ADAMS software can not only describe suspension parts, steering system components and their connections in detail, but also consider flexibility of connections and local parts, making the vehicle model more approximate to a real car.

Of course, the car is a very complex mechanical system, establishing the model as the actual structure of vehicle will be a huge task and is very difficult to realize. Therefore, in the process of modeling, appropriate abstraction and simplification was done to the vehicle according to the study 
purpose [8]. The following assumptions on the vehicle dynamics model are made in the paper.

(1) Power drive system are simplified, just consider the power transmission after axle shaft, that means driving torque is directly added in the constant velocity universal joint;

(2) The flexible connection between rigid body are simplified, using linear (nonlinear) elastic sleeve to simulate dynamic characteristics of actual conditions;

(3) Except the tires, damping elements, elastic elements and rubber components, other parts are regarded as rigid elements, and their deformation in simulation is ignored;

(4) Friction force in kinematics pairs is ignored.

Using Cartesian coordinates as vehicle coordinate system, while the vehicle is stationary on a level surface, the $\mathrm{x}$-axis is defined as parallel to the ground point to the forward direction, $\mathrm{z}$-axis is perpendicular to the ground point to the top and y-axis is the left side of the driver, the origin of the coordinate system is the intersection of the centerline of the wheel and forward surface of the car. The main motion parameters associated to handling stability and ride comfort are as following: angular velocity component of car body in the z-axis-the yaw rate, cars' centered speed component in the y-axis-Lateral Velocity "v", cars' centered acceleration component in the y-axis-Lateral acceleration ay, etc.

\subsection{Component Modeling}

First a template is established in ADAMS/Car, then the subsystems can be generated with templates, finally the model is generated by the subsystems, i.e. The subsystem is a combination of different templates, suspension and vehicle models are built on the basis of the various subsystems, modeling sequence as shown in Fig. (1).

A certain type of vehicle suspension system model is established in this paper, shown in Figs. $(2,3)$, the rest of the steering system model, tire model, engine model and braking system model are obtained by modifying the basic parameters of the model in ADAMS/Car system.

\subsection{Full Vehicle Model}

Assemble each subsystem that has been established in ADAMS/Car standard interface, we can get the full vehicle model we need. The assembly includes suspension and full vehicle that through simulation analysis [9].
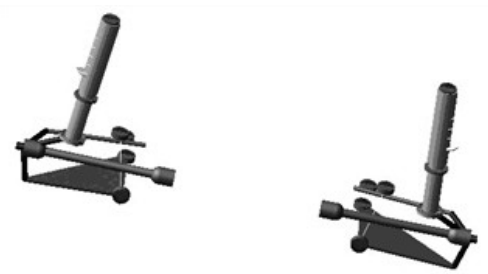

Fig. (2). The model of front suspension.
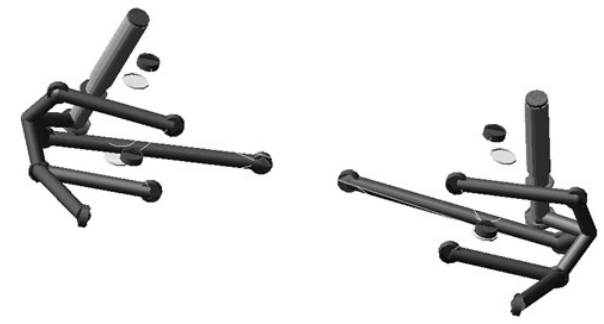

Fig. (3). The model of rare suspension.

The full vehicle assembly model includes : subsystem of front suspension, subsystem of front suspension stabilizer bar, subsystem of rear suspension, subsystem of power transmission, subsystem of steering, subsystem of breaking, subsystem of car body, lateral stabilizer bar, subsystem of front tire and rear tire. The subsystem models are assembled together, to get the dynamics model of the car, as shown in Fig. (4).

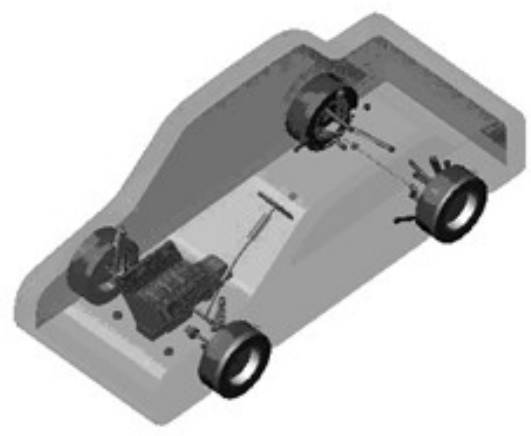

Fig. (4). Vehicle dynamics model.

\subsection{Model Validation}

In order to validate the vehicle dynamics model, the car needs to undertake steering stability test series under drypavement conditions. Take the driver's steering wheel control and speed control time history as input signal of the model in actual test, simulation analysis are carried out on the vehicle and the simulation results with the real test results are compared.

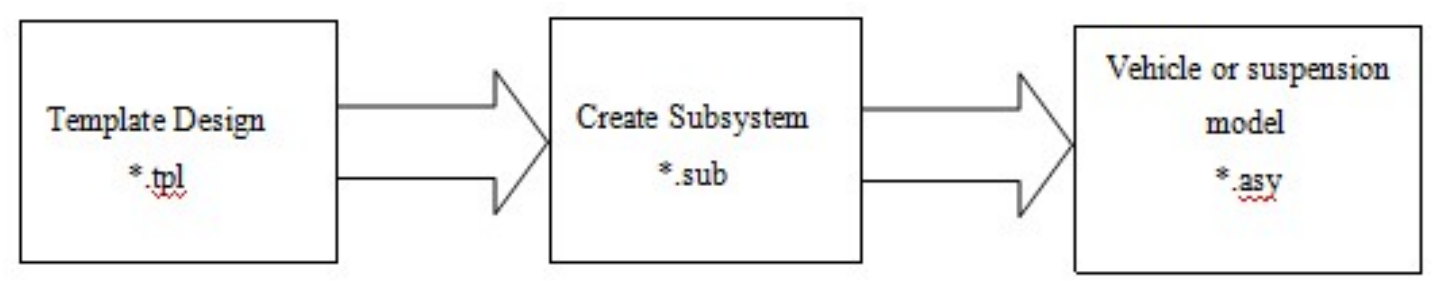

Fig. (1). The order of modeling. 
Fig. (5) is the contrast of dry pavement sine delay test according to the specifications provided by the factory with the simulation results. It can be seen that the simulation results are in good agreement with the real vehicle test results, it shows that the model accuracy is appropriate and the model can be used for simulation analysis of vehicle handling dynamics performance. The reason why the two cannot completely coincide maybe the models were corresponding simplified when establishing each system (For example, the steering system stiffness and damping characteristics, suspension system and the tires model simplification).

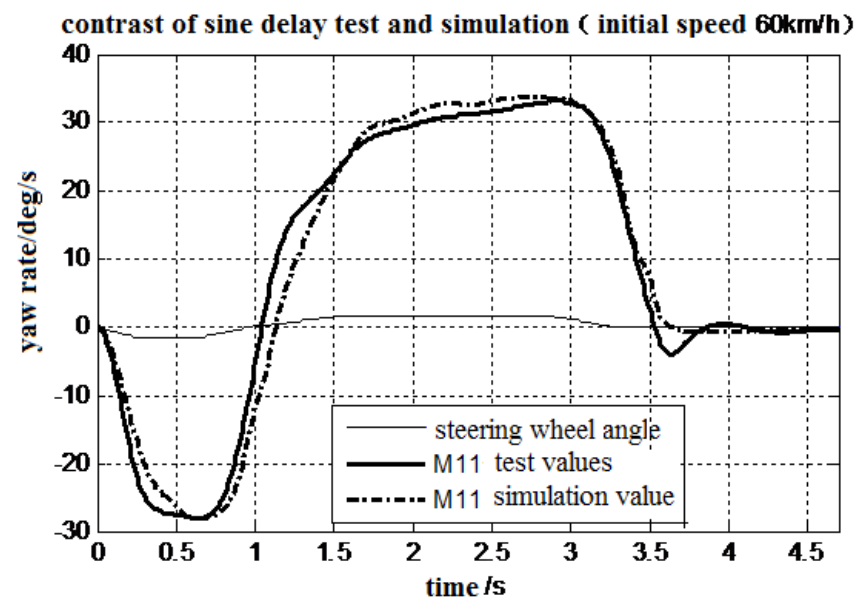

Fig. (5). The contrast of yaw rate in sine with dwell test.

\section{ESP CONTROL STRATEGY}

\subsection{Principle of Co-Simulation}

The principle of co-simulation is to establish a joint car model simulation system in ADAMS/Car and complete the joint simulation system control section in Matlab/Simulink, then connect these two simulation by ADAMS/Controls [10, 11]. Matlab and simulink controls output to improve the running state of the model of the vehicle, at the same time, yaw rate, sideslip angle and other parameters of ADAMS/Car are fed back to the control system model, so as to realize the interactive simulation in control system software environment, and to study the car dynamic simulation results in ADAMS/View or ADAMS/Solver.

Fig. (6) is the co-simulation schematic diagram of ESP with ADAMS/Car and Matlab/Simulink which take vehicle yaw rate and sideslip angle as control variable, that is, get the yaw rate and sideslip angle and other parameter from the virtual prototype model firstly, then compare with the desired yaw rate and the sideslip angle of the reference model to judge the stability state of vehicle and brake the corresponding wheel.

\subsection{ESP Control Strategy}

In the paper, the fuzzy control method is used, based on yaw rate, the controller module is the core of ESP joint simulation system and it is mainly to complete vehicle stability judgment and active yaw control [8], the internal structure as shown in Fig. (7).

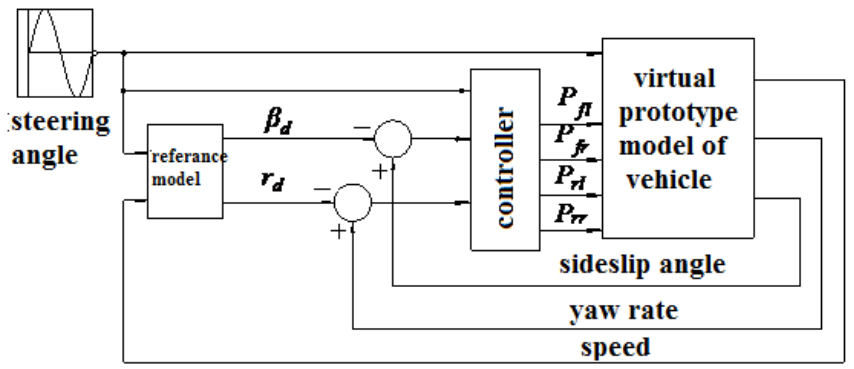

Fig. (6). The schematic diagram of co-simulation based on ADAMS/Car and Matlb/Simulink.

Stability control method which used in the vehicle mainly includes logic threshold control, PID control, optimal control, fuzzy control, neural networks, synovial control and robust control. Based on fuzzy math, fuzzy control method sets represent variables and advanced control strategy by fuzzy logic inference operation judgment.

Firstly, state variables were definited in ADAMS/CAR environment, the later velocity, longitude velocity and yaw velocity of vehicle were definited as the output state variable of vehicle dynamics model, and the steering wheel Angle was set as the input variable of vehicle multi-body dynamic model. Then the multi-body dynamics model of the vehicle were output to the Matlab environment in the form of a nonlinear controlled object, be represented as ADAMS _ sub subsystem automatically $[12,13]$. At last, the co-simulation model of the vehicle could be built, based on the ADAMS sub subsystem in the Matlab/Simulink environment. The vehicle dynamics state could be transported through the interface to the controller, then the controller issued the control instructions after logic operation, Control commands acted on the vehicle through the interface, so that the vehicle could run at a desired state kinetics.

According to ESP control theory, set yaw rate deviation $e(r)\left(e(r)=r-r_{\text {des }}\right)$ and its rate of change $e c\left(e c=\frac{d e(r)}{d t}\right)$ as input, output $U$ is yaw moment, fuzzy control theory based on the ESP yaw as shown in Fig. (8).

Set the fuzzy set of the e, ec and $\mathrm{U}$ are $\{\mathrm{NB}, \mathrm{NM}, \mathrm{NS}$, ZE, PS, PM, PB ; They belong to the domain of fuzzy set theory are $\{-6,-5,-4,-3,-2,-1,0,1,2,3,4,5,6\},\{-1,-0.8$, $0.6,-0.4,-0.2,0,0.2,0.4,0.6,0.8,1\},\{-7,-6,-5,-4,-3,-2,-$ $1,0,1,2,3,4,5,6,7\}$.

The membership functions, control rules are shown in Table 1. Among them, NB, NM, NS, ZE, PS, PM, PB represent Negative Bi, Negative MediumNegative Small, Zero, Positive Small, Positive Medium, Positive Big respectively.

\section{CONTROL STRATEGY VALIDATIONS}

In order to verify the accuracy of the simulation model, first the test series on dry pavement are applied to measure vehicle motion state, and get the vehicle steering angle input as drive files, then take them as inputs of ADAMS vehicle dynamics model and ADAMS/Matlab co-simulation model respectively to carry simulations and analysis stability of the ESP control system. 


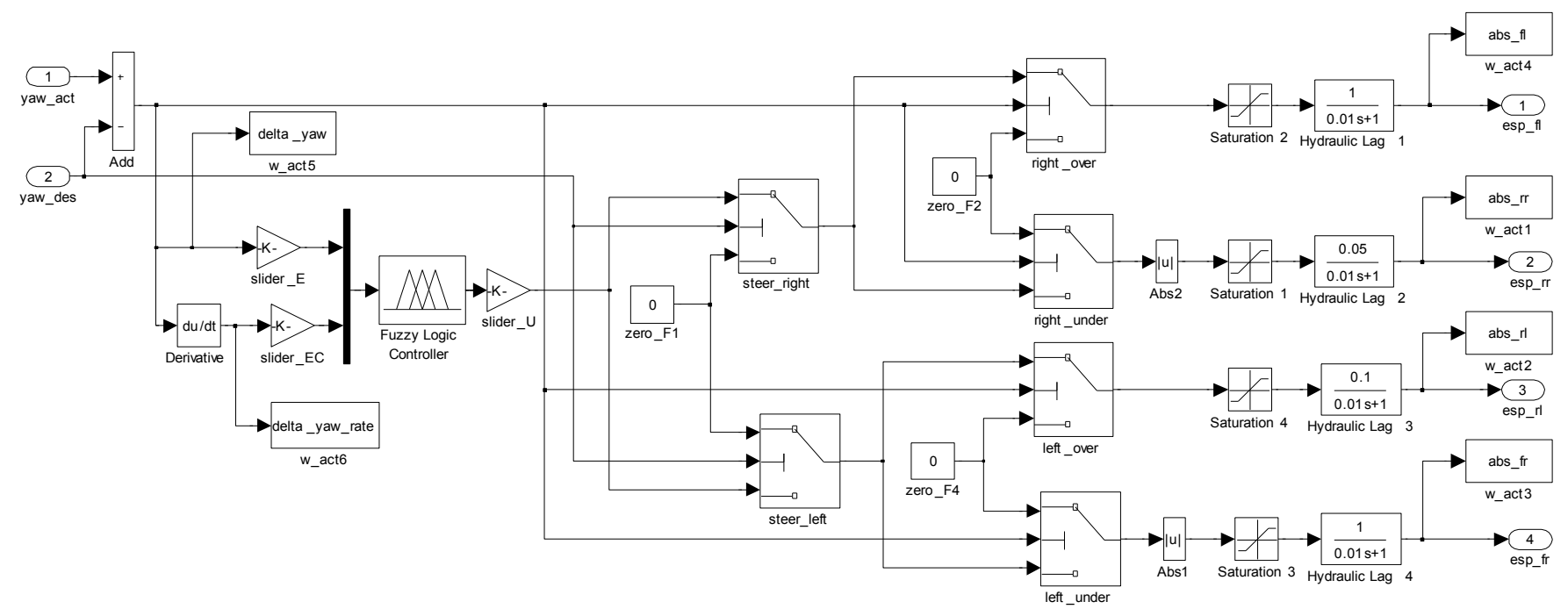

Fig. (7). Internal controller of ESP.

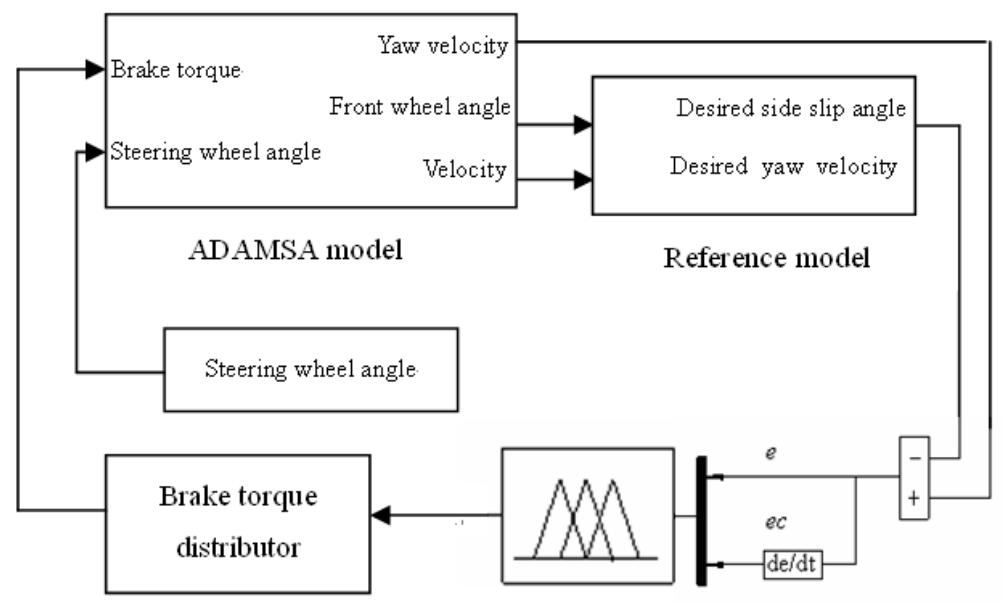

Fuzzy controller

Fig. (8). The fuzzy control principle.

Table 1. Control rules ( $\kappa$ is weight coefficients).

\begin{tabular}{|c|c|c|c|c|c|c|c|c|c|c|c|}
\hline $\boldsymbol{e}(\boldsymbol{\beta})$ & $\boldsymbol{e}(\boldsymbol{r})$ & $\boldsymbol{M}_{\boldsymbol{z}}$ & $\boldsymbol{\kappa}$ & $\boldsymbol{e}(\boldsymbol{\beta})$ & $\boldsymbol{e}(\boldsymbol{r})$ & $\boldsymbol{M}_{\boldsymbol{z}}$ & $\boldsymbol{\kappa}$ & $\boldsymbol{e}(\boldsymbol{\beta})$ & $\boldsymbol{e}(\boldsymbol{r})$ & $\boldsymbol{M}_{\boldsymbol{z}}$ & $\boldsymbol{\kappa}$ \\
\hline \hline NB & PB & NB & 1 & PB & PS & NS & 1 & ZE & NS & PS & 1 \\
\hline NS & PB & NB & 1 & NB & NB & PB & 1 & PS & NS & PM & 1 \\
\hline ZE & PB & NM & 1 & NS & NB & PB & 1 & PB & NS & PS & 1 \\
\hline PS & PB & NB & 1 & ZE & NB & PM & 1 & NB & ZE & NS & 1 \\
\hline PB & PB & NB & 1 & PS & NB & PB & 1 & NS & ZE & NS & 1 \\
\hline NB & PS & NB & 1 & PB & NB & PB & 1 & ZE & ZE & ZE & 1 \\
\hline NS & PS & NM & 1 & NB & NS & PB & 1 & PS & ZE & PS & 1 \\
\hline ZE & PS & NS & 1 & NS & NS & PM & 1 & PB & ZE & PS & 1 \\
\hline PS & PS & NM & 1 & & & & & & & & \\
\hline
\end{tabular}

\subsection{Dry Pavement Control Strategy Research}

Fig. (9) is a dry double lane road vehicle test which is carried out in accordance with ISO 3888-2 standard and simulation analysis of the curve, the initial speed was 65 $\mathrm{km} / \mathrm{h}$. It can be seen that when ESP was turn on, the vehicle could run according to the predetermined test path and kept stable state of motion, but when ESP was turn off, the vehicle yaw rate curve began to diverge at the moment of 2 $\mathrm{s}$, and vehicle could not maintain a stable state and was away from the test path.

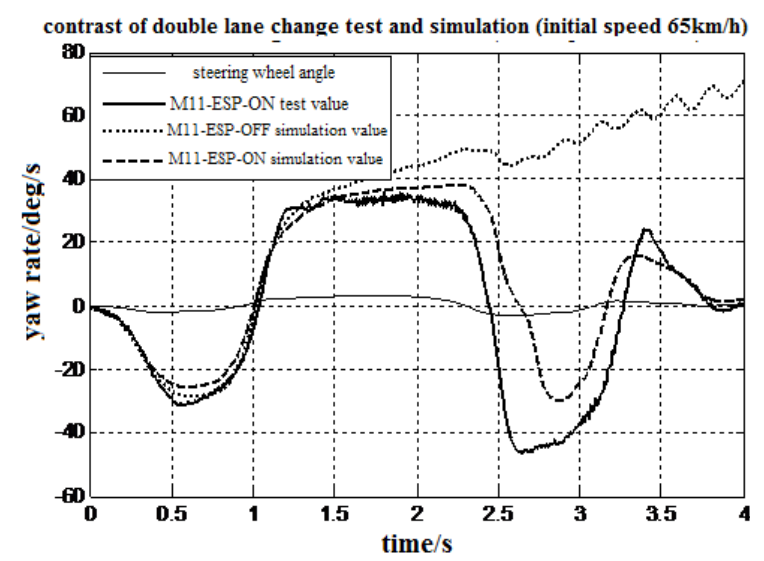

Fig. (9). The contrast of test and simulation in double lane test on dry road. 
Fig. (10) is a test and simulation analysis curve according to a company dry pavement sine delay specification, the initial speed was $80 \mathrm{~km} / \mathrm{h}$. It can be seen that the vehicle test curve meets well with the simulation curve, when ESP was turn off, the vehicle started instability at $1.5 \mathrm{~s}$ and cannot run according to the predetermined test path, while when ESP was turn on, the vehicle could run according to the predetermined test path and kept steady state.

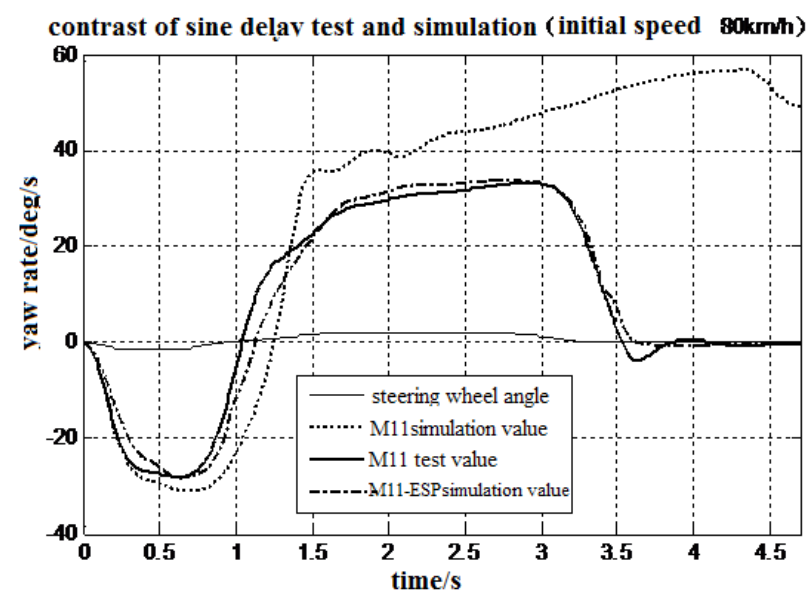

Fig. (10). The contrast of test and simulation in sine with dwell on dry road.

\subsection{Simulation of Control Strategy on Snow and Ice Road}

Take steering wheel angle which obtained by dry pavement double lane change test as input and modify tireroad friction coefficient to 0.1 in road file, then make simulation of double lane change test on ice road, the results are shown in Fig. (11). It can be seen that, the vehicle could keep steady state of motion on ice road when ESP was turn off, but the vehicle motion parameters vary considerably when around the second pile and there was trend of losing stability. While the vehicle motion parameters changed little and could run according to predetermined test path when ESP is turn on. Fig. (11a) is contrast of yaw rate, when ESP was turn on it changed slowly, the maximum value was about $0.7 \mathrm{deg} / \mathrm{s}$, while ESP was off the maximum value of yaw rate was about $3 \mathrm{deg}$. Vehicle lateral acceleration value as shown in Fig. (11b), the maximum value are respectively $0.08 \mathrm{~g}$ and $0.025 \mathrm{~g}$ when ESP was on/off. Fig. (11c) is contrast of side slip angle. It can be seen that when ESP was off, side slip angle changed greatly and the maximum value was about $-0.13 \mathrm{deg}$, while when ESP was on, the maximum value of side slip angle was about $-0.05 \mathrm{~g}$, the vehicle could finish the test according to predetermined path. Fig. (11d) is contrast of roll angle, when ESP was off the vehicle roll angle maximum value was $0.08 \mathrm{deg}$, while when ESP was on the maximum value of vehicle roll angle was $0.025 \mathrm{deg}$ and could keep steady state of motion.

Fig. (12) shows contrast of yaw rate, lateral acceleration, sideslip angle and roll angle which obtained by simulation of ice road state, the input was dry pavement sine delay test data. It can be seen the vehicle did not loss steering stability, but in the first second its motion parameters changed largely, (a) contrast of yaw rate

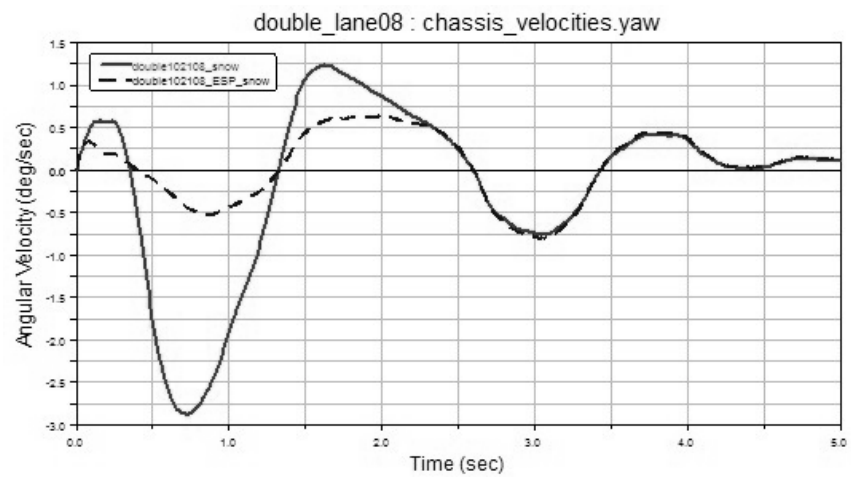

(b) contrast of lateral acceleration

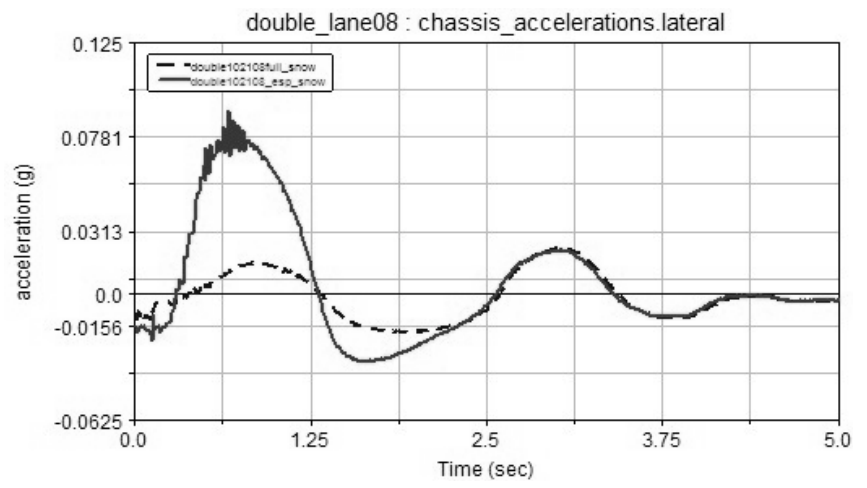

(c) contrast of side slip angle

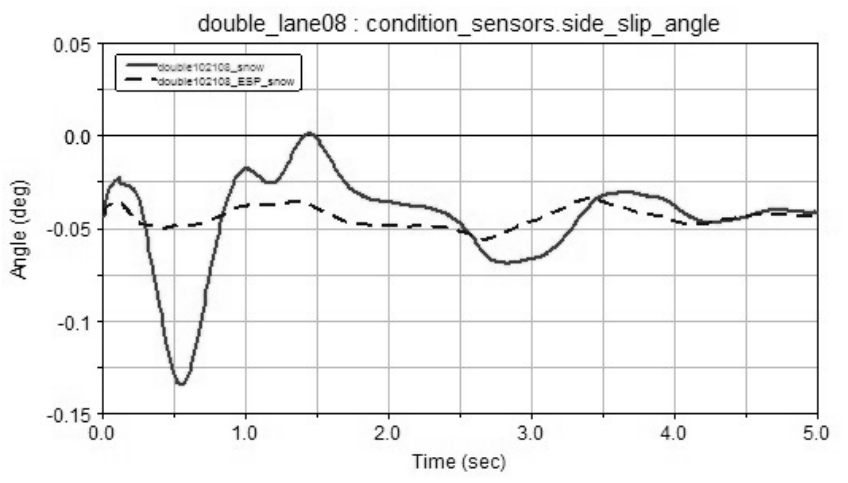

(d) contrast of vehicle roll angle

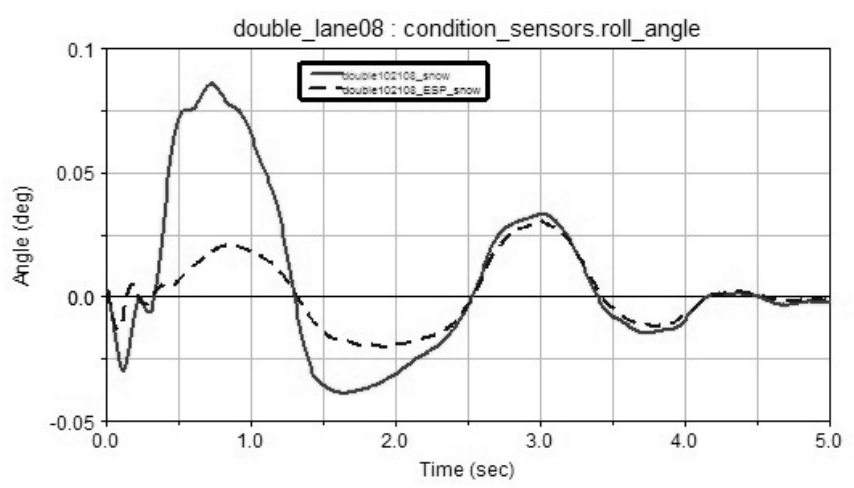

Fig. (11). Simulation of double lane test on ice road.

it tended to stable state after that. When ESP was off, the maximum value of yaw rate reached $-2.5 \mathrm{deg} / \mathrm{s}$, lateral acceleration, sideslip angle and roll angle reached $0.07 \mathrm{~g}$, 
$-0.15 \mathrm{deg}$ and $0.1 \mathrm{deg}$ respectively, the maximum value of vehicle parameters were decreased and the vehicle could keep steady state while ESP was on.

(a) contrast of yaw rate

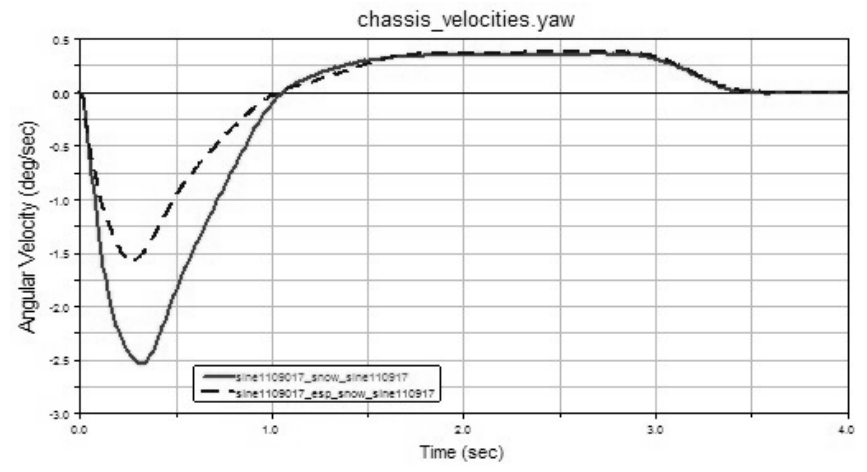

(b) contrast of latera 1 acceleration

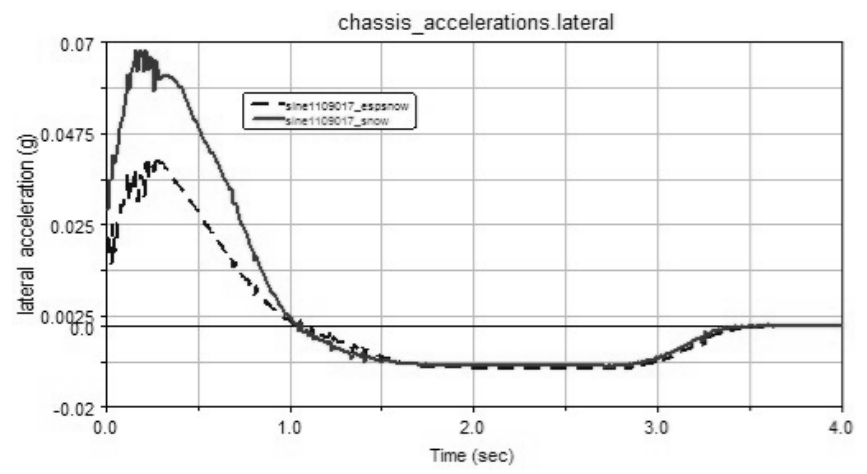

(c) contrast of side slip angle

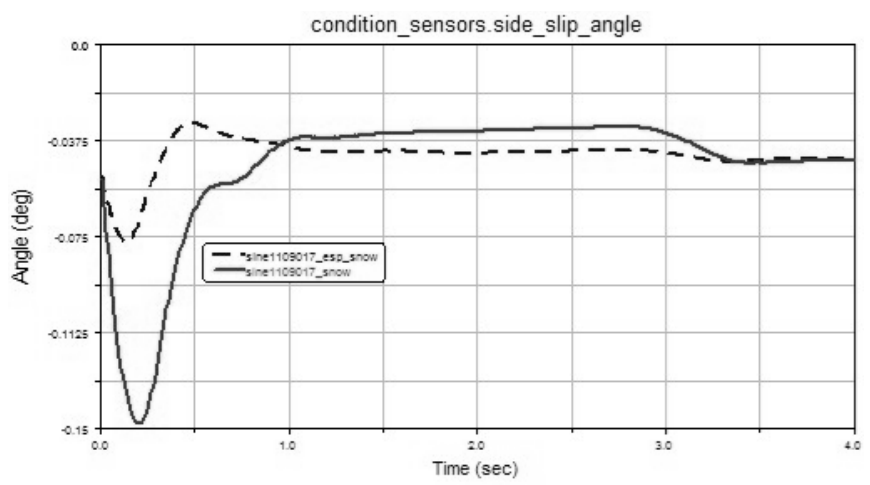

(d) contrast of roll angle

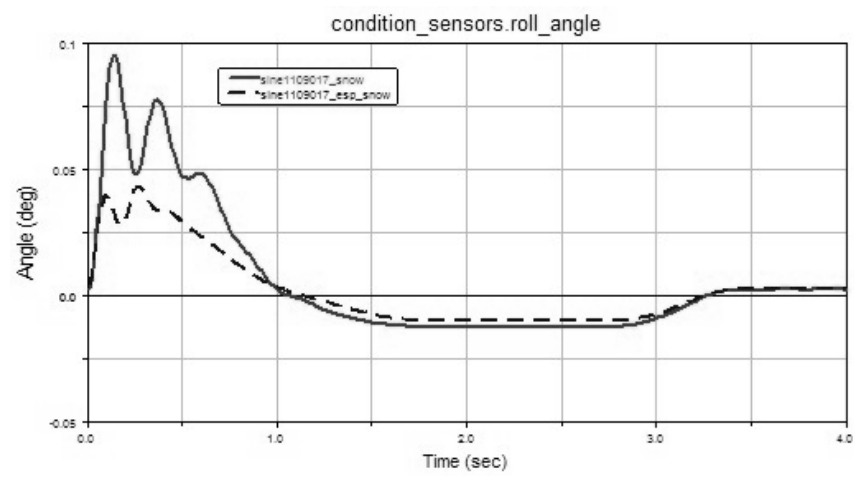

Fig. (12). Simulation of sine with dwell on ice road.

\section{CONCLUSION}

(1) On the basis of ADAMS system dynamics model, set the input and output variables and establish ADAMS/MATLAB co-simulation model;

(2) Established a fuzzy control model which take yaw rate as control variable to simulate the vehicle ESP control system;

(3) Compared the curves of dry pavement sine delay test and the simulation curves, the vehicle system dynamics model was supposed to be able to simulate and analyze the ESP control performance;

(4) Take the steering wheel angle data obtained by dry pavement test as driver file input, and set the tire-road friction coefficient to conduct a series of simulation tests of ice road. The results of simulation showed that the ESP control strategy set in this paper could effectively maintain stability of the vehicle and improve steering stability.

\section{CONFLICT OF INTEREST}

The authors confirm that this article content has no conflict of interest.

\section{ACKNOWLEDGEMENTS}

This work was supported by the National Natural Science Foundation of China (Grant Nos. 51205055) and the Fundamental Research Funds for the Central Universities (Grant Nos. DL13CB07).

\section{REFERENCES}

[1] J. Kim, "Effect of vehicle model on the estimation of lateral vehicle dynamics", Int. J. Automot. Technol., vol. 11, no. 3, pp. 331-337, 2010.

[2] Y. E. Papelis, T. Brown, G. Watson, D. Holtz, W. Pan, "Study of ESC Assisted Driver Performance Using a Driving Simulator", National Adv. Driving Simulat. Document ID: N04-003-PR.

[3] N. Giessen, Current Analysis of the Accident Statistics: Mercedes Passenger Cars Get Into Fewer Accidents. November 26, 2002.

[4] M. Aga, and A. Okada, "Analysis of vehicle stability control (VSC)'s effectiveness from accident data," In: Proce. $18^{\text {th }}$ Int. Tech. Conf. Enhanced Safety of Veh, 2003.

[5] Federal Motor Vehicle Safety Standards: Electronic stability control systems, controls and displays. National Highway Traffic Safety Administration: 49 CFR Parts 571 and 585, Docket No. NHTSA-200727662, 2007.

[6] H. Leffler, R. Auffhammer, R. Heyken, H. Röth, "New Driving Stability Control System with Reduced Technical Effort for Compact and Medium Class Passenger Cars", SAE Paper No: 980234, 1998.

[7] H. Fennel, E. L. Ding, "A Model-Based Failsafe System for the Continental TEVES Electronic-Stability-Program (ESP)", SAE Paper No: 2000-01-1635, 2000

[8] G. Tan, "Intelligent PID controller based on ant system algorithm and fuzzy inference and its application to bionic artificial leg", $J$. Central South Univ. Technol., vol. 11, no. 3, pp. 316-322, 2004.

[9] B. Ozdalyan, "Development of a slip control anti-lock braking system model", Int. J. Automot. Technol., vol. 9, no. 1, pp. 71-80, 2008.

[10] Y. Xu, "A study on vehicle rollover avoidance control based on active steering technique", Automot. Eng., vol. 5, no. 27, pp. 518$521,2005$. 
[11] T. Yan, K. Ren, Y. Wang, L. Zhang, "Fuzzy hybrid control strategy of semi-active suspensions for vehicle", Trans. Chin. Soc. Agric. Mach., vol. 43, no. 12, pp. 11-15, 2012.

[12] F. Yang, Y. Li, J. Ruan, X. Rong, and R. Song, "Real-time estimation of tire road friction coefficient based on extend state observer", Trans. Chin. Soc. Agric. Mach., vol. 41, no. 8, pp. 6-9, 2010.

[13] S. Li, L. He, "Co-simulation study of vehicle ESP system based on ADAMS”, J. Wuhan Univ. Technol. (Transport. Sci. Eng.), vol. 36, no. 6, pp. 1143-1147, 2012.

(C) Li et al.; Licensee Bentham Open.

This is an open access article licensed under the terms of the Creative Commons Attribution Non-Commercial License (http://creativecommons.org/licenses/by-nc/3.0/) which permits unrestricted, non-commercial use, distribution and reproduction in any medium, provided the work is properly cited. 